\title{
The number of waves on unstable vortex rings
}

\author{
By P. G. SAFFMAN \\ Applied Mathematics, California Institute of Technology, Pasadena
}

(Received 19 Mry 1977)

An explanation is proposed for the dependence on Reynolds number and other parameters of the number of waves which appear on vortex rings formed by pushing fluid out of a tube. It is shown that the number of waves can be sensitive to the vorticity distribution in the core of the ring. The process of ring formation is discussed and it is concluded that peaked vorticity distributions, limited by viscosity, will occur. Quantitative estimates of the number of waves are made. Agreement with observation is satisfactory.

\section{Introduction}

The instability of vortex rings formed by pushing fluid out of a tube was convincingly demonstrated by Krutzsch (1939), who presents remarkable pictures of the phenomena and gives quantitative data. Subsequent workers (e.g. Widnall \& Sullivan 1973; Liess \& Didden 1976; Maxworthy 1972, 1977) have reported similar observations. The instability consists of the appearance and growth of azimuthal waves around the circumference of the ring, followed later by a rapid breakdown of this perturbation of the apparently laminar ring when the waves have reached a finite size. The waves may or may not be uniformly distributed around the ring (Krutzsch 1939) and the subsequent breakdown may also be localized initially. The breakdown mixes efficiently the tracers commonly used to make the ring visible, and after the breakdown is completed the ring appears to settle down into a fairly steady and uniform state, which seems to be turbulent. Krutzsch does, however, imply that a repeat of the instability, wave growth followed by breakdown, was very occasionally seen. The well-known cascading of buoyant vortex rings, easily produced, for example, by dropping a small drop of dye into a glass of water, is an example of a repeating instability of vortex rings (see Yuen (1973) for an analysis of this instability). But no other observer has reported seeing the Krutzsch instability repeat itself.

An intuitive but convincing explanation of the beginning of the instability, the formation of azimuthal waves, was given by Widnall, Bliss \& Tsai (1974). $\dagger$ The basic mechanism was confirmed by Moore \& Saffman (1975) and by Tsai \& Widnall (1976) for the related problem of a rectilinear line vortex in a uniform plane strain, and confirmed in detail for uniform thin-cored rings by Widnall \& Tsai (1977). The idea is that if a rectilinear line vortex can be deformed into a sinusoidal filament with wavelength $2 \pi / k$ which is steady under its own self-induced velocity field (i.e. crests do not rotate or translate), and is then placed in a uniform plane strain normal to the axis of the undisturbed vortex, the displaced portions of the filament are convected away from

+ This paper explains why the earlier explanation given by Widnall \& Sullivan (1973) is fallacious; see also Moore \& Saffman (1974). 
their undisturbed positions with velocities proportional to their displacement and hence the wave grows. A vortex ring with core radius small compared with the ring radius is like a rectilinear line vor tex in a uniform plane strain, this being the velocity field induced near a point on the ring by the rest of the ring, and can therefore be unstable to disturbances in which the axis of the core is deformed from a circle. The number of waves which appear is therefore $k R$, where $R$ is the radius of the ring and $k$ can be found from the analysis of steady infinitesimal waves on an isolated rectilinear vortex filament.

Of course, $k R$ must be an integer. This means that $k$ will not be exactly the wavenumber of the steady infinitesimal wave, but will be in the band of wavenumbers which exist around this wavenumber and are subject to the instability. The wavenumber and bandwidth scale inversely with the core radius, and suitable values exist for thin cores. Moreover, the general mathematical analysis of the instability (Moore \& Saffman 1975) shows that the necessary condition for instability is that the dispersion equation for infinitesimal waves on an isolated vortex is degenerate, i.e. two waves, with different angular dependence around the core but the same axial wavelength, have the same speed. If there is no axial velocity along the core, it follows from symmetry that there are two steady disturbances which bend the axis into helices of opposite sense, which can be superposed into a steady plane sinusoid, and this mode is always unstable in an external plane strain. If there is axial flow, the steady disturbance is not necessarily degenerate and may not give rise to the instability. This question has a bearing on the usual non-repeatability of the instability, since it has been observed (Maxworthy 1977) that breakdown creates axial flow and it is a natural suggestion (although as yet unsupported by analysis and left for further study) that axial flow suppresses the instability.

The purpose of the present note is to discuss the number of waves that occur in the first stage of the instability and compare theoretical predictions with the various observations that have been made. Widnall \& Tsai (1977), following their previous studies, performed calculations for uniform vortex rings without axial flow, that is for rings such that the vorticity is exactly constant in the corresponding rectilinear filament. Such a ring of radius $R$, core radius $a$ and strength $\Gamma$ moves with velocity

$$
U=\frac{\Gamma}{4 \pi R}\left(\ln \frac{8 R}{a}-\frac{1}{4}\right)
$$

There is an enumerable infinity of axial wavelengths for steady sinusoidal disturbances on the corresponding filament, depending on how many nodes there are in the velocity distribution of the perturbation. For the two lowest modes with one or two nodes, $k a=2 \cdot 5$ and $4 \cdot 35$, respectively. Then for the lowest mode, which is usually assumed to be the one most likely to occur, the number of waves $N$ on the vortex ring would be given approximately by

where

$$
\begin{gathered}
N=k R=0 \cdot 40 e^{\tilde{U}}, \\
\tilde{U}=4 \pi U R / \Gamma .
\end{gathered}
$$

In the experiments of Liess \& Didden (1976), $N$ increased from 4 to 11 , while $\bar{O}$ remained constant with the value 3. Krutzsch (1939) and even more so Maxworthy (1977) found a significant dependence of $N$ upon Reynolds number. Maxworthy reported $N$ increasing from 10 to nearly 30 when the Reynolds number increased by a factor of 10. The experiments by Krutzseh and Maxworthy do not directly contradict (1.2) as 
the values of $\bar{U}$ were not actually measured and must be inferred from the given data, but plausible models of the mechanies of vortex-ring formation (see $\S 3$ ) suggest that the dependence of $\widehat{U}$ un Reynolds number is weak and that the data are therefore not consistent with (1.2).

One possible explanation of the discrepancy between theory and observation lies in the assumption that the core of the vortex is uniform. In the past few years, ideas on vortex formation put forward by Kaden and others more than 40 years ago have been rediscovered and make clear that the vorticity distribution in the core may be far from uniform. It is likely to be strongly peaked inside an inner core and then decay algebraically until it drops to zero at the ill-defined edge of the core. We shall now explore the consequences of this possibility, and show that it explains the experimental observations.

\section{Dependence of wavenumber on velocity profile}

Let us consider a rectilinear axisymmetric vortex filament with a continuous tangential velocity field $V(r)$ and no axial flow. The strength of the vorticity is

$$
\omega=r^{-1} d(V r) / d r
$$

We shall suppose that the vorticity extends only a finite distance from the axis, i.e. $\omega=0$ when $r>a$. The strength of the vortex is

$$
\Gamma=\int_{0}^{a} 2 \pi r \omega d r=2 \pi a V(a) .
$$

For $r>a$, the velocity field is that of a potential vortex, i.e.

$$
V=\Gamma / 2 \pi r .
$$

The velocity vanishes on the axis if $\omega$ is finite there.

In addition to the core radius $a$, there are two other length scales of significance. First, there is the 'inner core radius' $a_{1}$, at which $V$ has its maximum. Clearly $a_{1} \leqslant a$. Second, there is the 'effective core radius' $a_{e}$, related to the translational speed of the vortex ring formed by bending a long segment of the filament into a circle of radius $R$, where $R \gg a$. The speed of the ring is given by (1.1) when $\omega$ is constant. When $\omega$ is not constant, it was shown by Fraenkel (1970) and Saffman (1970) that, with an error $O\left(a^{2} / R^{2}\right)$

$$
L=\frac{\Gamma}{4 \pi R}\left(\ln \frac{8 R}{a}-\frac{1}{2}+Z\right)
$$

where

$$
Z=\frac{4 \pi^{2}}{\Gamma^{2}} \int_{0}^{a} r V^{2} d r
$$

Saffman (1970) also shuwed that this result remains valid when the velocity field in the core is changing with time cwing to viscous diffusion. For a uniform distribution of vorticity, $Z=\frac{1}{4}$. The effective core radius is defined by

$$
a_{e}=a e^{t-Z}, \text { or } a_{e}=8 R e^{-(\tilde{U}+1)} \text {. }
$$

It has the property that the speed of the ring is given by (1.1) when $a$ is replaced by $a_{\ell}$.

$$
\dagger \text { Factors that are a power of } \ln (R / a) \text { are here regarded as } O(1) \text {. }
$$


Other length scales can of course be defined, such as the radius of gyration of the vorticity distribution, but these three, $a, a_{1}$ and $a_{e}$, seem to be the ones with the most physical significance. Of course, $a$ will not in practice be well defined, if only becanse departures from axisymmetry will increase with distance from the core.

We now consider the axial wavelength of infinitesimal three-dimensional perturbations which are unstable when the vortex has been slightly deformed by a weak steady irrotational plane strain with streamlines perpendicular to the axis of the vortex. As discussed in $\S 1$, unstable disturbances are related to steady modes with angular dependence $e^{ \pm i \theta}$ and the dispersion equation for waves on the undeformed vortex provides an equation for the unstable axial wavelengths as functionals of the velocity distribution in the vortex. There is in general an enumerable infinity of such axial wavelengths, depending on the radial structure inside the vortex of the steady mode, i.e. on the number of radial nodes. Each wavelength is also the centre of a narrow band of unstable wavelengths, the width of the band being proportional to $\epsilon$, the magnitude of the extension rate in the plane strain. The maximum amplification rate in a band occurs at the centre and is proportional to $\epsilon$.

There are other unsteady modes which can be degenerate and correspond to instability. For instance, the right-handed helical mode with $n$ nodes can have for some axial wavelength the same non-zero frequency as a left-handed helical mode with $n^{\prime}$ nodes; or a helical mode with angular dependence $e^{i \theta}$ may have the same frequency as a triangular fluted mode with angular dependence $e^{3 i \theta}$. However, in contrast to the situation for the steady modes, the wavelengths arising from the unsteady degeneracies do not necessarily correspond to instability of the deformed vortex. Each case has to be treated on its merits. Tsai \& Widnall (1976) calculated some cases for the vortex with uniform core, and found that when unstable the growth rates of the unsteady degeneracies were an order of magnitude less than those for the steady modes. This is in accord with Widnall, Bliss \& Tsai's physical mechanism as described above in $\$ 1$, and we shall take this as justification for neglecting the unsteady modes and concentrating on the steady ones. In addition the calculation is thereby made much simpler, as it is far easier to solve the eigenvalue problem for the axial wavenumber given the frequency than vice versa.

It is convenient to introduce dimensionless variables defined by

$$
s=\frac{r}{a}, \quad \Omega=\frac{a}{r} \frac{V(r)}{V(a)} .
$$

The steady infinitesimal perturbations of the radial, tangential and axial velocity and the pressure, of the form $v_{r}(s) E, v_{\theta}(s) E, v_{z}(s) E$ and $p(s) E$, where $E=e^{i(k \varepsilon+m \theta)}$, are easily seen to satisfy

$$
\left(\begin{array}{cccc}
i m \Omega, & -2 \Omega, & 0, & d / d s \\
\Delta, & i m \Omega, & 0, & i m / s \\
0, & 0, & i m \Omega, & i \kappa \\
d / d s+1 / s, & i m / s, & i \kappa, & 0
\end{array}\right)\left(\begin{array}{c}
v_{r} \\
v_{\theta} \\
v_{z} \\
p
\end{array}\right)=0
$$

where

$$
\Delta=2 \Omega+s \Omega^{\prime}
$$

and the prime denotes $d / d s$. The velocity profile of the undisturbed vortex fixes $\Omega$. The 
requirement that (2.8) has a non-trivial solution vanishing at $s=\infty$ and regular at $s=0$ determines the dimensionless axial wavenumber $\kappa=k a$ as an eigenvalue of the system. Only the values $m= \pm 1$ are of interest here.

If we define two new independent variables by

$$
y_{1}=i v_{r}, \quad y_{2}=p / \Omega,
$$

the system $(2.8)$ reduces to

$$
\left.\begin{array}{l}
y_{1}^{\prime}=\left(\frac{\Delta}{\Omega}-1\right) \frac{y_{1}}{s}-\left(\kappa^{2}+\frac{1}{s^{2}}\right) y_{2}, \\
y_{2}^{\prime}=\left(\frac{2 \Delta}{\Omega}-1\right) y_{1}-\frac{\Delta}{\Omega} \frac{y_{2}}{s} .
\end{array}\right\}
$$

For solid-body rotation, $\Delta / \Omega=2$. This is also the value at the centre of the vortex if the vorticity is finite. For $s>1, \Delta / \Omega=0$. Continuity of velocity and pressure requires that $y_{1}$ and $y_{2}$ are continuous across a discontinuity of $\Delta$. (The variables in $(2.10)$ are not convenient if the vortex has a stagnant core, where $\Omega=0$. Continuity of $y_{1}$ and $y_{2}$ will fail if there are vortex sheets in the distribution, across which $\Omega$ is discontinuous. It is better to treat such cases separately.)

For $s>1$, the solution is

$$
y_{1}=A \kappa K_{1}^{\prime}(\kappa s), \quad y_{2}=-A K_{1}(\kappa s),
$$

where $A$ is an arbitrary constant. For $s<1$, we define $\left(z_{1}, z_{2}\right)$ to be the solution of (2.11) satisfying the regularity conditions

$$
\begin{gathered}
z_{1} \rightarrow 1, \quad z_{2} \sim s \quad \text { as } \quad s \rightarrow 0 \\
y_{1}=B z_{1}, \quad y_{2}=B z_{2},
\end{gathered}
$$

where $B$ is an arbitrary constant. The eigenvalue problem is to determine $\kappa$ such that

$$
\kappa K_{1}^{\prime}(\kappa) / K_{1}(\kappa)=z_{1}(1) / z_{2}(1) .
$$

For given $\Omega$, the right-hand side of $(2.15)$ can be calculated numerically and the search for real values of $\kappa$ which are roots of (2.15) is straightforward.

It is now necessary to choose a suitable class of rotation profiles $\Omega(s)$. For reasons to be discussed in $\$ 3$, a profile which admits the inclusion of viscous effects into vortex rings produced by apparatus for which there exists an extensive body of data is the one with

$$
\Omega(s)=M\left(\frac{3}{4} ; 2 ;-s^{2} / \epsilon^{2}\right) / M\left(\frac{3}{4} ; 2 ;-1 / \epsilon^{2}\right), \quad s \leqslant 1 .
$$

$M(a ; b ; z)$ is the confluent hypergeometric function in the notation of Abramowitz \& Stegun (1965, p. 503); it has the asymptotic property that

$$
M(a ; b ; x) \sim[\Gamma(b) / \Gamma(b-a)](-x)^{-a} \quad \text { as } \quad x \rightarrow-\infty .
$$

The number $\epsilon^{2}$ is inversely proportional to a Reynolds number. For this profile, $s_{1}=a_{1} / a, \gamma_{1}=s_{1}^{2} \Omega\left(s_{1}\right)$ and $s_{e}=a_{e} / a$ are given to a good approximation by

$$
s_{1}=1 \cdot 4 \tilde{\sigma} \epsilon, \quad \gamma_{1}=\epsilon^{\frac{1}{2}}, \quad s_{e}=0 \cdot 47+0 \cdot 63 \epsilon
$$




\begin{tabular}{|c|c|c|c|}
\hline$\epsilon$ & $\kappa_{1} s_{1}$ & $\kappa_{2} s_{1}$ & $\kappa_{3} s_{1}$ \\
\hline$\infty$ & $2 \cdot 51$ & $4 \cdot 35$ & $6 \cdot 17$ \\
\hline 5 & $2 \cdot 51$ & $4 \cdot 36$ & $6 \cdot 20$ \\
\hline 1 & 2.74 & $4 \cdot 76$ & 6.75 \\
\hline 0.9 & 2.79 & $4 \cdot 86$ & 6.89 \\
\hline $0 \cdot 8$ & $2 \cdot 88$ & $5 \cdot 01$ & $7 \cdot 10$ \\
\hline 0.7 & $3 \cdot 01$ & $5 \cdot 22$ & $7 \cdot 40$ \\
\hline 0.6 & $2 \cdot 79$ & $4 \cdot 84$ & $6 \cdot 84$ \\
\hline 0.5 & $2 \cdot 56$ & $4 \cdot 44$ & $6 \cdot 25$ \\
\hline 0.4 & $2 \cdot 37$ & $4 \cdot 09$ & $5 \cdot 74$ \\
\hline 0.3 & $2 \cdot 19$ & $3 \cdot 78$ & $5 \cdot 29$ \\
\hline 0.2 & $2 \cdot 02$ & $3 \cdot 45$ & $4 \cdot 78$ \\
\hline $0 \cdot 1$ & $1 \cdot 81$ & $2 \cdot 97$ & $4 \cdot 06$ \\
\hline 0.05 & 1.65 & $2 \cdot 42$ & 3.59 \\
\hline
\end{tabular}

TaBle 1. Product of wavenumber and inner core radius for the lowest three modes. Rotation profile given by $(2.16)$.

for $\epsilon<0 \cdot 5$. Here $\gamma_{1}=\Gamma_{1} / \Gamma$, where $\Gamma_{1}$ is the circulation at the radius of maximum tangential velocity.

The vorticity profile given by (2.16) is strongly peaked at the centre as $\epsilon \rightarrow 0$, and possesses a well-defined inner core. (It is also the appropriate profile for the laminar trailing vortices produced by an elliptically loaded wing; see Moore \& Saffman 1973.) The uniform core is recovered in the limit $\epsilon \rightarrow \infty$.

With this profile we have calculated in the manner just described the dimensionless wavenumbers $\kappa_{1}, \kappa_{2}$ and $\kappa_{3}$ of the first three modes. Values of $\kappa_{1} s_{1}, \kappa_{2} s_{1}$ and $\kappa_{3} s_{1}$ are shown in table 1 . (Note that for $\epsilon>0.69, s_{1}=1$.)

Professor D. W. Moore has pointed out that the differential equations (2.11) can be solved analytically, in terms of Bessel functions, for the profile $\Omega=\epsilon^{-\frac{3}{2}}, s \leqslant \epsilon ; \Omega=s^{-\frac{3}{2}}$, $\epsilon \leqslant s \leqslant 1$. The results are similar, and provided a useful check on the numerical method for calculating the $\kappa$ 's. The values of $\kappa$ for the model profile are the roots of

$$
J_{2}\left(\kappa \epsilon 3^{\frac{1}{2}}\right)+3^{\frac{1}{2}} J_{1}\left(\kappa \epsilon 3^{\frac{1}{2}}\right)\left[K_{0}(\kappa) / K_{1}(\kappa)+\kappa(1-\epsilon)\right]=0 .
$$

As $\epsilon \rightarrow 0$, the values of $\kappa \epsilon 3^{\frac{1}{2}}$ approach the roots of $J_{1}$.

The number of waves on a ring of radius $R$ is

$$
N \approx \frac{\kappa R}{a}=\frac{1}{8} \kappa s_{1} \frac{s_{e}}{s_{1}} e^{\widetilde{U}+\frac{1}{4}}
$$

The quantities $\kappa s_{1}$ and $s_{e} / s_{1}$ are functions only of the shape of the velocity profile. $\kappa s_{1}$ according to table 1 varies relatively slowly; $s_{e} / s_{1}$ varies much more rapidly as shown by (2.17). The variation of $N$ with Reynolds number for given $\tilde{U}$ is consistent with $(2.18)$ if the core structure is sensitive to viscous effects for large Reynolds numbers. This is to be expected; for instance, Moore \& Saffman (1973) showed that the cores of laminar trailing vortices produced by lifting wings at large Reynolds number are controlled by viscous mechanisms. We now consider how the vortex-ring core depends upon viscosity. 


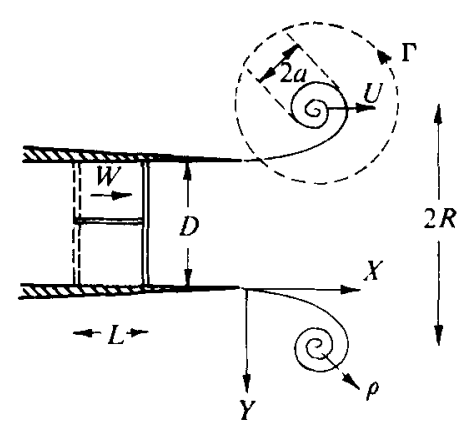

(a)

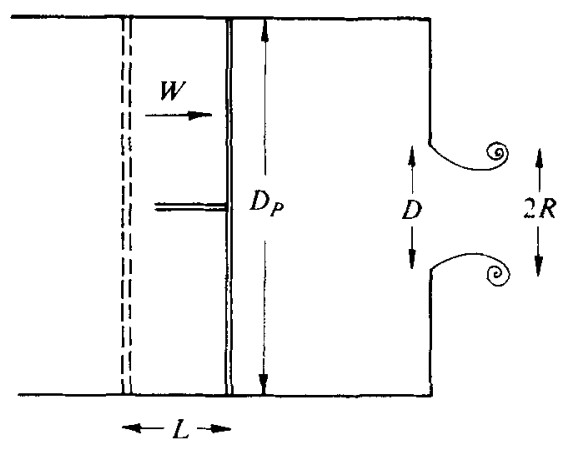

(b)

FIGURE 1. Sketch of common geometries used in the production of vortex rings.

\section{The vortex-ring structure}

Vortex rings are commonly produced by pushing fluid out of a tube or through a hole; see figure 1. The flow separates at the sharp edge of the hole, in order to satisfy a Kutta condition, and a vortex sheet forms which rolls up into a vortex ring. Even in a perfect fluid, the calculation of the formation and evolution of the vortex sheet is a hard problem. In real fluids, further complications are caused by the velocity induced by the vortex sheets producing boundary layers on the outside surfaces of the tube which separate and make vorticity and vortex sheets of the opposite sign. Here we shall just discuss some simple qualitative ideas.

The quantities of interest are the radius of the ring $R$, its circulation $\Gamma$ and the vorticity distribution in the core. The latter will determine the dimensionless scales $s_{e}$ and $s_{1}$. When the Reynolds number, defined by $R e=W D / \nu$, is large compared with one, it can be expected that the effect of viscosity on the formation and development of the vortex sheet is small and that its main effect is to remove a singular, non-analytic behaviour of the velocity at the centre of the spiral. Then on dimensional grounds,

$$
\frac{2 R}{D}=f\left(\frac{L}{D}, \frac{D}{D_{P}}\right), \quad \frac{\Gamma}{W L}=g\left(\frac{L}{D}, \frac{D}{D_{P}}\right) .
$$

Here $L$ is the stroke, $W$ the piston speed and $D / D_{P}$ the ratio of hole diameter to piston diameter. Of course, the unknown functions $f$ and $g$ will depend upon the precise way in which the piston is pushed, that is on the variation of $W$ with time. Crude theories have been formulated (Saffman 1975; Linden \& Turner, unpublished; Maxworthy 1977) to predict their properties, but they are not entirely satisfactory. Two points should be kept in mind. First, if $L / D$ is too small, the vortex ring is produced close to the tube and, instead of propagating away, moves back into the tube under the induced velocity of its image in the tube walls. Sheffield $(1977 a)$ has calculated the critical distances for a vortex pair to move into or away from the ends of a channel, for the corresponding two-dimensional problem. Second, if $L / D$ is too large, the vortex sheet will break up into a train of vortex rings.

The result ( $3.1 \mathrm{a}$ ) appears to be reasonably consistent with the data published by Krutzsch (1939), Liess \& Didden (1976) and Maxworthy (1977) for apparatus of type $(a)$, and by Sallet \& Widmayer (1974) for type (b) apparatus. Krutzsch's data do, 
however, show considerable scatter compared with the others; it is possible that he did not control the piston motion sufficiently carefully. A weak dependence on Reynolds number can be seen in some data, the value of $R$ increasing slightly with the Reynolds number.

The relation $(3.1 b)$ is harder to confirm, since circulation is difficult to measure and $\Gamma_{1}$ can be confused with $\Gamma$, if a uniform core is assumed in the analysis of the data. Liess \& Didden's data provide the best support; they found that $\Gamma / W L$ was constant at about 0.7 for $L / D=1.4$ and Reynolds numbers of 2000-10000. Maxworthy's results also seem to show Reynolds number independence; they also suggest that $\Gamma / W L$ decreases as $L / D$ increases, which is qualitatively consistent with some simple theoretical ideas, which will now be described.

When the fluid has just been set into motion, and the vortex sheet is of small extent and close to the edge, it behaves like a two-dimensional vortex sheet formed at the edge of a semi-infinite flat plate. As discussed by Anton (1939) and Wedermeyer (1961) (see also Kulkarny 1977), there is a similarity solution for this flow akin to the one described by Kaden (1931) for the roll-up of a semi-infinite vortex sheet. Suppose that the flat plate extends along the $-x$ axis. Then at $t=0$, the velocity potential is $\phi=\alpha r^{\frac{1}{2}} \cos \frac{1}{2} \theta$, using polar co-ordinates centred on the edge of the plate. The value of $\alpha$ is determined by matching to the flow far from the edge and $\alpha / W D^{\frac{1}{2}}$ is a function of the geometry; e.g. a piston moving with velocity $W$ down a two-dimensional channel of width $D$ gives $\alpha=W(D / \pi)^{\frac{1}{2}}$. For small times, the vortex sheet which appears at the edge, in order to satisfy the Kutta condition, and which rolls up into a spiral, depends only on $\alpha$ and $t$. Its centre $(X, Y)$ (see figure 1), the circulation $\Gamma(\rho)$ about a circle of radius $\rho$ centred on the vertex of the spiral, and the total circulation $\Gamma$ shed from the edge depend on $\rho, t$ and $\alpha$ as follows by dimensional analysis:

$$
X=c_{1}(\alpha t)^{\frac{2}{3}}, \quad Y=c_{2}(\alpha t)^{\frac{2}{3}}, \quad \Gamma(\rho)=c_{3} \alpha \rho^{\frac{1}{2}}, \quad \Gamma=c_{4} \alpha^{\frac{4}{3}} t^{\frac{1}{3}} .
$$

The numbers $c_{1}, c_{2}, c_{3}$ and $c_{4}$ are constants. (Estimates $c_{1}=0.09, c_{2}=0.3, c_{3}=3.3$ and $c_{4}=2 \cdot 5$ can be obtained from Anton and Wedermeyer's calculations.) The expressions (3.2) fail because either the piston stops moving when $t=L / W$ or the spiral has become comparable in size to the diameter of the hole when $t=c_{5} D / W$, where $c_{5}$ is another unknown constant depending upon the geometry; it should be different for apparatus of type $(a)$ and $(b)$.

If the former time is less than the latter (i.e. $L<c_{5} D$ ), we can substitute $t=L / W$ into (3.2) to obtain estimates of the ring diameter $2 R$, core radius $a$ and total circulation $\Gamma$ for the resulting ring. We obtain

$$
\begin{gathered}
2 R=D+c_{2} c_{6}^{\frac{2}{3}} L^{\frac{2}{3}} D^{\frac{1}{3}}, \quad a=\left(c_{4}^{2} c_{6}^{\frac{2}{3}} / c_{3}^{2}\right) L^{\frac{2}{3}} D^{\frac{1}{3}}, \\
\Gamma=c_{4} c_{6}^{\frac{4}{3}} W L^{\frac{1}{3}} D^{\frac{2}{3}},
\end{gathered}
$$

where $c_{6}=\alpha / W D^{\frac{1}{2}}$. The rotation profile in the core is

$$
\Omega(s)=s^{-\frac{3}{2}} .
$$

The estimates (3.3) ignore the interaction of the ring with the walls after the piston has stopped (see Sheffield $1977 a$ ). This may be significant for small $L / D$, leading to a decrease in $R$ below that given by (3.3a). Krutzsch's data show $2 R<D$ for $L / D<\frac{1}{2}$. 
Otherwise, (3.3 $a$ ) fits the data of Krutzsch, Liess \& Didden and Maxworthy reasonably well up to $L / D=2$ with $c_{2} c_{6}^{\frac{2}{3}}=0 \cdot 22$. The value of $\Gamma$ agrees with Liess \& Didden's measurements if $c_{\mathbf{4}} c_{\mathbf{6}}^{\frac{4}{3}}=0 \cdot 86$. The decrease in $\Gamma / W L$ with increasing $L / D$ reported by Maxworthy is in qualitative agreement with $(3.3 \mathrm{c})$, but the predicted values with the stated value of the constant are about $25 \%$ too high. $\dagger$ There are insufficient data available for type (b) apparatus to make a comparison.

The velocity profile associated with (3.4) is singular at the centre of the spiral. The velocity decreases monotonically from infinity at $s=0$. The singularity is removed by viscosity; see Kirde (1962) or Moore \& Saffman (1973). If $t$ is the time from the start of the piston motion, and $(4 \nu t)^{\frac{1}{2}} \ll a$, where $\nu$ is the kinematic viscosity, the action of viscosity changes (3.4) into the hypergeometric-function profile given by (2.16), with

$$
\epsilon^{2}=4 \nu t / a^{2} \text {. }
$$

An estimate of the constant in (3.3b) can be obtained from the measured speed of vortex rings. We have

$$
\widetilde{O}=\ln (8 R / a)-\frac{1}{4}-\ln \varepsilon_{e^{r}}
$$

Now $s_{e}$ is a function of $\epsilon$ [see (2.17)] and hence also of $t$. Rings will not therefore move with constant speed, but should slowly decelerate. Alternatively, the speed at a fixed distance depends on the Reynolds number. However, the dependence is weak. Taking $c_{4}^{2} c_{6}^{\frac{2}{3}} / c_{3}^{2}=0 \cdot 28$, we obtain $V^{0}$ varying between $2 \cdot 8$ and $3 \cdot 0$ for Liess \& Didden's experiment; they reported a constant value $\tilde{U}=2 \cdot 9$. (The estimates taken from Anton (1939) and Wedemeyer (1961), together with $c_{6}=\pi^{-\frac{1}{2}}$, give $c_{2} c_{6}^{\frac{2}{3}}=0 \cdot 2, c_{4} c_{6}^{\frac{4}{3}}=1 \cdot 1$ and $c_{4}^{2} c_{6}^{2} / c_{3}^{2}=0 \cdot 4$. The agreement with values deduced from the data is reasonable, especially when it is remembered that the calculations are for plane flow.)

The expression (3.6) for the speed certainly fails after the instability has progressed through transition or breakdown to turbulence. The faster deceleration which then occurs can be explained only by a loss of vorticity to a wake, probably caused by turbulent mixing: see Maxworthy (1974). Since $a / R=0.4$ for $L / D=1.4$ according to $(3.3 a, b)$ with the above constants, the diffusion of vorticity into a wake should be negligible for a laminar ring and (3.6) should be an adequate approximation until transition occurs.

\section{The number of waves on an unstable vortex ring}

We now consider how many waves appear on the vortex ring when it goes unstable. The analysis described in $\$ 2$ is strictly valid only for steady, inviscid vortices. The properties of real laminar vortex rings should be time dependent. However, as seen for the speed, the time variation is slow and we can make the customary assumption that the growth rate of the instability is large in comparison, and hence treat the instability as occurring on a profile frozen at time $T$, say. Let us now suppose that the properties of vortices produced in apparatus of type $(a)$ are as just described. In particular, the profile is given by (2.16) and $\epsilon$ by (3.5). We can express equation (2.18) for $N$ as

$$
N \approx \kappa s_{1} R / a_{1}=0 \cdot 34 \kappa s_{1} R(\nu T)^{-\frac{1}{2}},
$$

† 'The assumption that the fluid comes out as a straight plug of length $L$ gives $\Gamma=\frac{1}{2} W L$, the factor $\frac{1}{2}$ arising because the flux of vorticity in the boundary layer is $\frac{1}{2} W^{2}$ (Kulkarny 1977). 


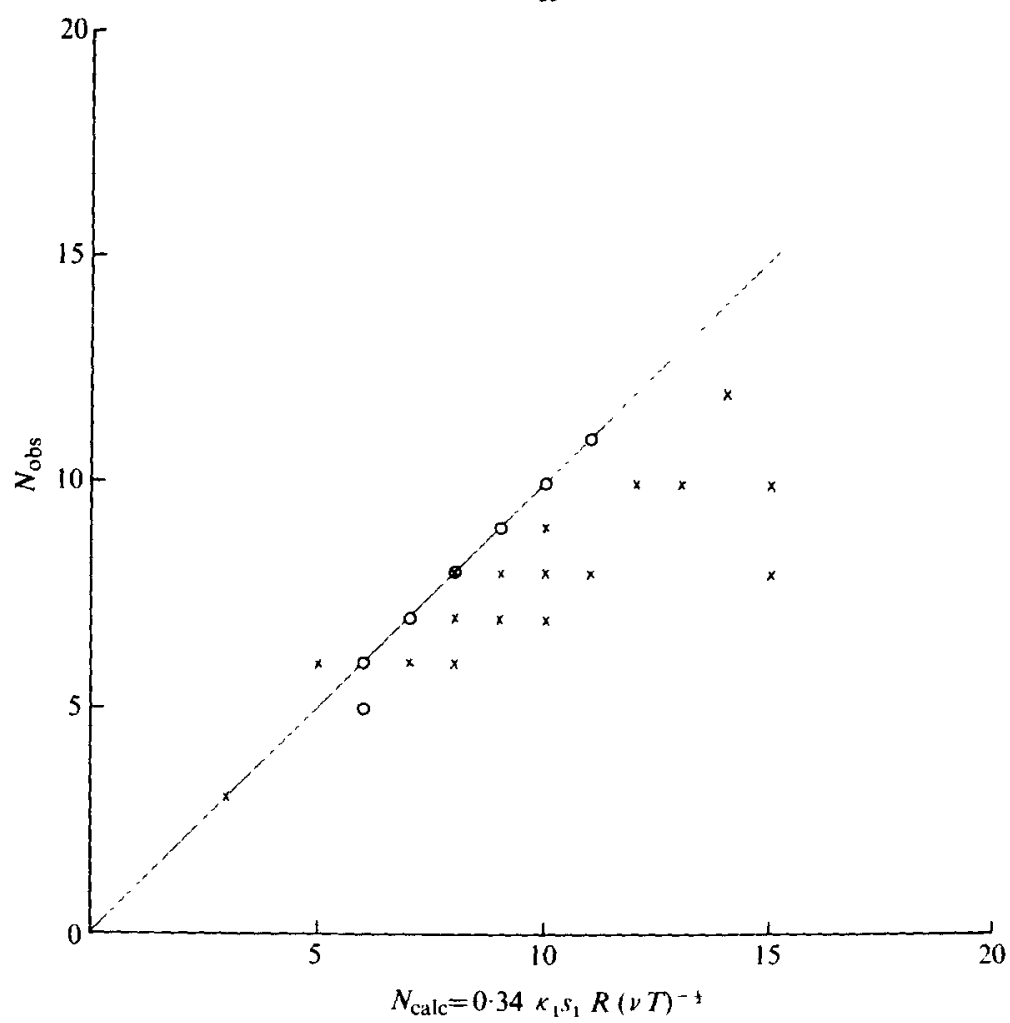

Figure 2. Comparison of calculated and observed values of $N$. Observed values: $x$, Krutzsch (1939); $O$, Liess $\&$ Didden (1976). Calculated values from (4.1).

where $\kappa s_{1}$ is listed in table 1 as a function of $\epsilon$. The value of $a$ is supposed to be given by $(3.3 b)$. Both Krutzsch and Liess \& Didden list values of $N, R, T, D$ and $L$, so that direct comparison can be made. Figure 2 shows values of $N$ calculated from (4.1), rounded to the nearest integer, plotted against the observed values. For the Liess \& Didden data, the agreement is excellent; for the Krutzsch data, the trend is favourable but the calculated number consistently exceeds the number observed, sometimes by a significant amount. In this comparison, $\kappa$ was identified with $\kappa_{1}$, i.e. the disturbance with the least internal structure and longest axial wavelength is supposed to occur. The number of waves predicted for higher modes of instability is greater, and the agreement would be worse.

Direct comparison with Maxworthy's data is not possible, as values of $R$ and $T$ are not given. However, they can be inferred if we make the plausible assumption that in a real fluid the instability occurs only when a critical Reynolds number is exceeded. It seems appropriate to base the Reynolds number on the inner core radius $a_{1}$ and the rate of strain $\sigma$ to which the core is subjected and which deforms it from a circular shape. Dyson (1893) gives the cross-sectional shape of a uniform vortex ring. Combining this with the results of Moore \& Saffman (1971) on the deformation of line vortices in uniform strain, we infer that

$$
\sigma=\frac{3 \Gamma}{16 \pi R^{2}}\left[\ln \frac{8 R}{a_{e}}-\frac{17}{12}\right]
$$


is the effective rate of strain to which the core is exposed. We assume that this expression holds for non-uniform cores. $\dagger$ If $c_{7}$ is the critical Reynolds number, we make the hypothesis that waves occur when

$$
\frac{\sigma a_{1}^{2}}{\nu}=c_{7}, \quad \text { i.e. } \quad T=\frac{c_{7}}{8.7} \frac{R}{U} \frac{\ln \left(8 R / a_{e}\right)-\frac{1}{4}}{\ln \left(8 R / a_{e}\right)-\frac{17}{12}} .
$$

The Liess \& Didden data are consistent with (4.3) if we take $c_{\eta}=60$. The Krutzsch data show considerable scatter, but (4.3) gives a fair fit. Krutzsch commented that there was a significant dependence on external disturbances and his results were not repeatable from run to run.

Maxworthy (1977) presents $N$ as a function of $R e=W D / \nu$. There is some ambiguity in the definition of $W$ since the piston speed is not exactly constant, as Maxworthy points out, and we shall simply use the average speed. From table 1, it can be seen that a reasonable approximation to $\kappa_{1} s_{1}$ is $1 \cdot 6+2 \epsilon$. Then using (3.3), we can write (4.1) as

$$
N \approx\left[0 \cdot 53+2 \cdot 43(D / L)^{\frac{2}{3}}\right][1+0 \cdot 8 / \epsilon] .
$$

To find $\epsilon$, we have to solve a transcendental equation, obtained from (4.3):

$$
\epsilon=18 \cdot 6 \frac{R}{a}\left(\frac{\nu}{\Gamma}\right)^{\frac{1}{2}}\left(\ln \frac{8 R}{a}-\ln s_{e}-\frac{17}{12}\right)^{-\frac{1}{2}}
$$

Using the results (3.3), we can express this in terms of $R e$ and $L / D$ :

$$
\begin{aligned}
\epsilon \operatorname{Re}^{\frac{1}{2}}=50\left(\frac{D}{L}\right)^{\frac{5}{8}}\left\{1+0.22\left(\frac{L}{D}\right)^{\frac{2}{3}}\right\}\left\{1 \cdot 24+\frac{2}{3} \ln \frac{D}{L}+\ln \left[1+0 \cdot 22\left(\frac{L}{D}\right)^{\frac{2}{3}}\right]\right. & -\ln (0.47+0 \cdot 63 \epsilon)\}^{-\frac{1}{2}} .
\end{aligned}
$$

From (4.4) and (4.6), we obtain $N$ as a function of $R e$ and $L / D$. Note that all constants in this equation are determined by the Liess \& Didden data. Agreement or otherwise with Maxworthy's data at higher Reynolds numbers therefore provides a test of the ideas.

Figure 3 contains a plot of $N v s$. Re for $L / D=1 \cdot 4$ as predicted by (4.4) and (4.6). The values of $N$ decrease very slightly as $L / D$ increases, $R e$ being kept fixed. The agreement with Maxworthy's data is remarkably close. $f$ The Liess \& Didden data, for $R e<10^{4}$, are not shown on this figure as the various constants were in fact so chosen that the calculated curve agrees with them.

\section{The growth rate}

An estimate can be made of the initial growth rate of the wave disturbance from results for a straight vortex in a uniform strain. The curvature of the vortex ring is thereby neglected, but Widnall \& Tsai's (1977) results show that this is not significant for a uniform-cored ring, and there is no reason to suspect that the situation would be

$f$ It is to be noted that $a_{e}$ can be expressed in terms of an equivalent cut-off for the BiotSavart law of self-induced motion of a vortex filament.

$\ddagger$ There appears to be some disagroement for $3 \times 10^{4}<R e<5 \times 10^{4}$, where a 'bimodal instability' is seen to occur. See $\$ 5$. 


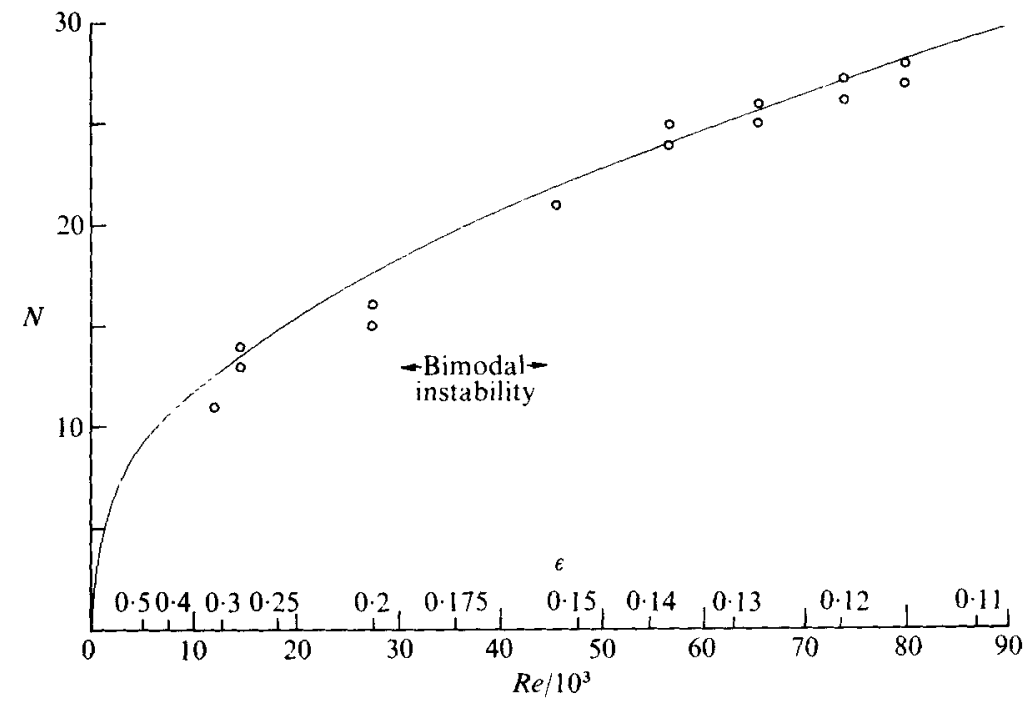

Figure 3. Dependence of number of waves on Reynolds number. Two numbers may coexist experimentally in the region called 'bimodal instability'. Values of $\epsilon$ are also shown. $O$, Maxworthy (1977); - calculated with $L / D=1 \cdot 4$.

$\begin{array}{ccccccc}\epsilon & P_{1} & 2 \pi a Q_{1} / \Gamma & P_{2} & 2 \pi a Q_{2} / \Gamma & P_{3} & 2 \pi a Q_{3} / \Gamma \\ \infty & 1.142 & 0.27 & 1.139 & 0.16 & 1.136 & 0.12 \\ 5 & 1.145 & 0.27 & 1 \cdot 143 & 0.16 & 1.140 & 0.12 \\ 1 & 1.220 & 0.27 & 1.217 & 0.17 & 1.213 & 0.12 \\ 0.9 & 1.234 & 0.27 & 1.231 & 0.17 & 1.227 & 0.12 \\ 0.8 & 1.252 & 0.27 & 1.248 & 0.17 & 1.245 & 0 \cdot 12 \\ 0.7 & 1.274 & 0.26 & 1.271 & 0.17 & 1.267 & 0.12 \\ 0.6 & 1.303 & 0.26 & 1.300 & 0.16 & 1.297 & 0.12 \\ 0.5 & 1.341 & 0.25 & 1.340 & 0.15 & 1.338 & 0.11 \\ 0.4 & 1.392 & 0.22 & 1.397 & 0.13 & 1.396 & 0.092 \\ 0.3 & 1.465 & 0.18 & 1.475 & 0.094 & 1.476 & 0.061 \\ 0.2 & 1.563 & 0.11 & 0.566 & 0.045 & 1.558 & 0.026 \\ 0.1 & 1.670 & 0.034 & 1.646 & 0.009 & 1.623 & 0.006 \\ 0.05 & 1.718 & 0.007 & 1.684 & 0.002 & 1.653 & 0.001\end{array}$

Table 2. Values of $P$ and $Q$, from which the growth rate and bandwidth of unstable disturbances on a strained rectilinear vortex can be obtained, for the rotation profile (2.16).

any different for the non-uniform cores of our study. The growth rate of a disturbance with axial wavenumber $k$ is

$$
\left[\sigma^{2} P^{2}-\left(k-k_{c}\right)^{2} Q^{2}\right]^{\frac{1}{2}},
$$

where $\sigma$ is the rate of strain (supposed small compared with $\Gamma / a^{2}$ and given approximately by (4.2) for a vortex ring) and $k_{c}$ is the axial wavenumber for a steady helical disturbance. It is supposed that $k$ is close to $k_{c}$. $P$ is a number and $Q$ is a velocity both of which depend on the velocity profile in the core and the structure of the steady disturbance. They are matrix coefficients of the steady eigenfunctions; the formulae are given by Moore \& Saffman (1975). (There is a misprint in equation (3.8) of the justquoted paper, a factor $\frac{1}{2}$ should multiply the matrix on the right-hand side.) 


\begin{tabular}{|c|c|c|c|c|}
\hline$\epsilon$ & $s_{e}$ & $\kappa_{1}$ & $\kappa_{2}$ & $\kappa_{3}$ \\
\hline 1 & 0.96 & 2.64 & $4 \cdot 60$ & $6 \cdot 52$ \\
\hline 0.9 & 0.95 & $2 \cdot 67$ & $4 \cdot 65$ & $6 \cdot 60$ \\
\hline 0.8 & 0.94 & $2 \cdot 71$ & $4 \cdot 72$ & $6 \cdot 70$ \\
\hline $0 \cdot 7$ & 0.93 & $2 \cdot 76$ & $4 \cdot 82$ & $6 \cdot 84$ \\
\hline $0 \cdot 6$ & 0.91 & $2 \cdot 84$ & $4 \cdot 96$ & $7 \cdot 04$ \\
\hline $0 \cdot 5$ & 0.89 & $2 \cdot 94$ & $5 \cdot 15$ & $7 \cdot 31$ \\
\hline 0.4 & 0.86 & 3.08 & $5 \cdot 40$ & $7 \cdot 68$ \\
\hline 0.3 & 0.84 & $3 \cdot 21$ & $5 \cdot 72$ & $8 \cdot 16$ \\
\hline 0.2 & 0.81 & $3 \cdot 28$ & $6 \cdot 11$ & $8 \cdot 75$ \\
\hline 0.1 & 0.79 & $3 \cdot 24$ & $6 \cdot 38$ & $9 \cdot 41$ \\
\hline
\end{tabular}

Values of $a k_{c}$ for the core profile given by $(2.16)$ can be obtained from table 1 for the first three modes. Values of $P$ and $2 \pi a Q / \Gamma$ for the same profiles and modes have been calculated by Sheffield (1977b) and are listed in table 2 . The growth rate is not unduly sensitive to the Reynolds number. The bandwidth increases as the inner core radius decreases.

The case $\epsilon=\infty$ is solid-body rotation, for which growth rates and bandwidths were calculated by Tsai \& Widnall (1976) for the lowest two modes. If their results are put into the present notation, it is found that $P_{1}=1 \cdot 142$ and $P_{2}=1 \cdot 139$, in agreement with those given here. The values of $Q$ also agree.

There are, of course, infinitely many unstable modes, each with a different value of $N$. The usual assumption is that the one with the largest growth rate is the one which occurs. The maximum growth rate is $\sigma P$ when $k=k_{c}$. For a uniform core in solid-body rotation, the lowest mode appears to be the most unstable, as found by Tsai \& Widnall (1976). But as $\epsilon$ decreases and Re increases, it appears from table 2 that there is a crossover at about $\epsilon \doteqdot \mathbf{0 . 5}, R e \doteqdot 5000$, and the lowest mode ceases to be the most unstable. However, for $\epsilon<0 \cdot 2, R e>30000$, the lowest mode again becomes the most unstable.

The successful comparison with experiment described in $\$ 4$ used the lowest mode. The explanation must lie in the values of $P$ being close together for the modes, so that the actual choice of unstable mode depends on effects neglected in the analysis. The most plausible candidate is viscosity, which is expected to damp high-order modes, with more internal structure, at a greater rate than the lowest-order mode, with least structure. Support for this view is provided by the success of (4.3) in correlating the time at which the instability appears. $\dagger$ If the growth rate is $\sigma P$, the change in $\epsilon$ during the growth of the instability is $O\left(R e^{-\frac{1}{2}}\right)$, and the neglect of the variation with time of the undisturbed state is justified.

The value of $k-k_{c}$ for instability must lie between $\pm \sigma P / Q$. Hence the value of $N$ must lie in the range

$$
k_{c} R-\sigma P R / Q<N<k_{c} R+\sigma P R / Q .
$$

The values of $\sigma P R / Q$ for the experiments are always greater than 0.5 , and hence integer $N$ always exist.

† It may also explain why the instability does not usually repeat. Turbulence produces a dissipation to damp the growth mechanism. 
One significant disagreement between theory and experiment remains to be mentioned. Maxworthy (1977) found a bimodal instability in the range

$$
3 \times 10^{4}<\operatorname{Re}<5 \times 10^{4},
$$

which he refers to as a new type of instability. Disturbances with a shorter (about onehalf) wavelength appear and waves of the two wavelengths are seen to exist at the same time. It is tempting to interpret Maxworthy's observations as the temporary occurrence of the second mode. The corresponding range of $\epsilon$ is $0 \cdot 2-0 \cdot 15$, and reference to table 1 shows that the number of waves of the second mode is approximately $70 \%$ greater. However, the detailed description of Maxworthy's observations does not fit this view; see for example his figure 12. Moreover, according to table 2 the range of $\epsilon$ for which the second mode is more unstable is $0 \cdot 5-0 \cdot 2$, so that the theoretical and experimental ranges do not overlap.

Finally, we should like to present a speculation about the dependence of $N$ upon $R e$ for experiments conducted in apparatus of type $(b)$. Saffman (1975) presented a model of vortex-ring formation based on the assumption that the fluid pushed out by the piston forms a cylindrical vortex sheet. This assumption is untenable for type (a) apparatus, but may be more reasonable for type $(b)$ because the constant $c_{5}$ [see paragraph following (3.2)] may be small and the spiral moves away from the hole before the piston stops and trails a cylindrical vortex sheet. A satisfactory comparison between theory and experiment was made only with type (b) apparatus. If this is so, the velocity profile in the final ring is not the same. In the inviscid ring, $\Omega(s)=s^{-1}$ is the appropriate distribution and

$$
\Omega=M\left(\frac{1}{2}, 2,-s^{2} / \epsilon^{2}\right) / M\left(\frac{1}{2}, 2,-1 / \epsilon^{2}\right)
$$

is the profile with viscosity included. The feature of this profile is that $s_{1}=1$, i.e. there is no peak of the tangential velocity and in this sense there is no inner core, although the vorticity is still peaked at the axis. The values of $\kappa_{1}, \kappa_{2}$ and $\kappa_{3}$ are shown in table 3 . The interesting feature is that the wavelengths now depend only weakly on the Reynolds number. Thus the number of waves on the unstable ring increases far more slowly with Reynolds number for type $(b)$ apparatus than for type $(a)$, if the ideas are correct. At present there is no experimental evidence on this matter.

This work originated in a discussion with Professor D. W. Moore. Support was provided by the Energy Research Development Administration (AT 04-3-767) and the U.S. Army Research Office, Durham (DAHC 04-75-C-0009).

\section{REFERENCES}

Abramowitz, M. \& Stegun, I. A. 1965 Handbook of Mathematical Functions. Washington: Nat. Bur. Stand.

Anton, L. 1939 Ing. Arch. 10, 411.

Dyson, F. W. 1893 Phil. Trans. Roy. Soc. A 184, 1041.

Fraenkel, L. E. 1970 Proc. Roy. Soc. A 316, 29.

KADEN, H. 1931 Ing. Arch. 2, 140.

Krade, K. 1962 Ing. Areh. 31, 385.

Krutzsch, C. H. 1939 Ann. Phys. 35, 497.

Kulkarny, V. A. 1977 On vortex formation near sharp edges in impulsive flows. To appear. 
Limss, C. \& Didden, N. 1976 Z. angew. Math. Mech. 56, T206.

Maxworthy, T. 1972 J. Fluid Mech. 51, 15.

Maxworthy, T. 1974 J. Fluid Mech. 64, 227.

MaXworthy, T. 1977 J. Fluid Mech. 81, 465.

Moone, D. W. \& SAfrman, P. G. 1971 In Aircraft Wake Turbulence (ed. Olsen, Goldburg \& Rogers), p. 339. Plenum.

Moore, D. W. \& Saffman, P. G. 1973 Proc. Roy. Soc. A 333, 491.

Moore, D. W. \& Safrman, P. G. 1974 Proc. Roy. Soc. A 338, 535.

Moone, D. W. \& Saffman, P. G. 1975 Proc. Roy. Soc. A 346, 413.

Safrman, P. G. 1970 Stud. Appl. Math. 49, 371.

Saffman, P. G. 1975 Stud. Appl. Math. 54, 261.

Sallet, D. W. \& Wrdmayer, R. S. 1974 Z. Flugwiss. 22, 207.

Shefrield, J. S. 1977 a Phys. Fluids 20, 543.

SheFrteld, J.S. $1977 b$ Ph.D. thesis, California Institute of Technology.

TsaI, C.-Y. \& WIDNaIX, S. E. 1976 J. Fluid Mech. 73, 721.

Wedermeyer, E. 1961 Ing. Arch. 30, 187.

Widnale, S. E., Bliss, D. B. \& Tsai, C.-Y. 1974 J. Fluid Mech. 66, 35.

Widnall, S. E. \& Sullrvan, J. P. 1973 Proc. Roy. Soc. A 332, 335.

Widnalt, S. E. \& TsaI, C.-Y. 1977 The instability of the thin vortex ring of constant vorticity. To appear.

Yuen, H. 1973 Ph.D. thesis, California Institute of Technology. 
